American Journal of Applied Sciences 5 (6): 639-644, 2008

ISSN 1546-9239

(C) 2008 Science Publications

\title{
Enhancing P300 Component by Spectral Power Ratio Principal Components for a Single Trial Brain-Computer Interface
}

\author{
${ }^{1}$ S. Andrews, ${ }^{2}$ Ramaswamy Palaniappan, ${ }^{3}$ Andrew Teoh and ${ }^{4}$ Loo chu Kiong \\ ${ }^{1}$ Faculty of Information Science and Technology, Multimedia University, Malacca, Malaysia \\ ${ }^{2}$ Department of Computing and Electronic Systems, University of Essex, \\ Colchester, United Kingdom \\ ${ }^{3}$ Biometric Engineering Research Centre, Yonsei University, South Korea \\ ${ }^{4}$ Faculty of Engineering and Technology, Multimedia University, Malacca, Malaysia
}

\begin{abstract}
Here we present a novel approach to detect P300 wave in single trial Visual Event Related Potential (VERP) signals using improved principal component analysis to enable a faster braincomputer interface (BCI) design. In the process, the principal components (PCs) are selected using novel methods, namely spectral power ratio (SPR) and sandwich spectral power ratio (SSPR). We set out to assess the improved performances of our proposed methods, SPR and SSPR over standard PC selection methods like Kaiser and residual power for speller BCI design. Concluding, the P300 parameters extracted through our proposed SPR and SSPR methods showed improved detection of target characters in the speller BCI.
\end{abstract}

Key words: Brain-computer interface, principal components, $\mathrm{P} 300$, single trial, spectral power ratio

\section{INTRODUCTION}

Paralysed people can utilise Brain-Computer Interface $(\mathrm{BCI})$ designs to communicate with their external environments as it does not require any conventional muscle control. In general, BCI technologies that utilise the Electroencephalogram (EEG) signals could be segregated into four categories, namely those using: i) motor imagery ( $\mu$ and beta rhythms) ${ }^{[1]}$, ii) mental activities ${ }^{[2]}$, iii) slow cortical potentials $^{[3]}$ and iv) visual event related potential (VERP) signals ${ }^{[4,5]}$. The advantage of using the VERP based $\mathrm{BCI}$ is that it is easier for the users to adopt and does not require any significant prior training. VERP based $\mathrm{BCI}$ can be further divided into those using $\mathrm{P} 300^{[4]}$ and steady state $\operatorname{VERP}^{[5]}$.

At present, there are many improvements taking place in the constituent modules of this BCI such as in the stimulus paradigm, recording (hardware) protocols, signal processing algorithms and output formats for a wide variety of applications with several objectives like enhanced accuracy, quicker response and simpler usability. It is hoped that these improvements in BCI will move the usability of the systems from laboratory to real world and impart confidence to locked-in patients to communicate in simple words.
In this study here, we explore single trial analysis of VERP signals to enable a quicker response from the BCI system. Conventionally, the VERP signal analysis is carried out using ensemble averaging of signals captured in repeated trials ${ }^{[6]}$. In specific, VERP based speller BCI often use P300 parameters to distinguish the target (focused) characters from other characters (unfocused) on the screen. This P300 response feature is buried in the background EEG that is not related to the response of the target stimuli and averaging is required to reduce the effects of this background EEG. This causes unacceptable delays in the system response due to the numerous trials involved and further, suffers from the fact that there could be latency distortions (due to weak time-locking of P300) in the averaged signal. Single trial analysis of VERP signals eliminates this distortion problem and results in much faster system responses, hence quicker communication.

Principal Component Analysis (PCA) is a popular technique that has been used for the extraction of single trials of VERP signals ${ }^{[7]}$. The selection of Principal Components (PCs) is an important issue in PCA and in this study, we address this issue by proposing novel methods based on the spectral content of the PC, namely the Spectral Power Ratio method (SPR) and Sandwich Spectral Power Ratio (SSPR)

Corresponding Author: S. Andrews, Faculty of Information Science and Technology, Multimedia University, Malacca, Malaysia 
methods. The objective of these improved PC selection methods would be to enhance the performance of PCA; in particular for the extraction of P300 component. We then move on to show the improvement in performance for detection of the target characters in speller BCI using SPR and SSPR over the standard PC selection methods like Kaiser (KSR) and residual power $(\mathrm{RP})$.

\section{THE DATA}

We are utilizing all available paradigm data sets in our research, here in this paper we are presenting the data from the dataset IIb made available in the BCI Competition 2003 website: (<http://ida.first.fraunhofer. de/projects/bci/competition_ii/> were used here. A description of this dataset could be found from $<$ http://ida.first.fraunhofer.de/projects/bci/competition_i i/albany_desc/albany_desc_ii.html $>$.

Sixty-four electrode channels from an electro-cap were used to record the signals from the gel-smeared scalp of one subject. These collected signals were digitised at $240 \mathrm{~Hz}$. The subject's task was to focus the attention on a six by six matrix (as shown in Fig. 1) that consisted of alphanumeric characters formed in a matrix of six rows and six columns. The objective was to detect the focused target character when the rows and columns were intensified randomly for $100 \mathrm{~ms}$ with frequency of $5.7 \mathrm{~Hz}$. The time delay between two consecutive intensifications was $75 \mathrm{~ms}$. Row/column intensifications were randomised in blocks of 12 and set of these 12 intensifications would be considered as one trial. Two (i.e., the specific row and column) out of 12 trials would need to be identified to decide the focused character. The potentials in the brain evoked by the intensifications were recorded. The VERP responses for the rows and columns that contain the target character will be different from the other 10 responses, which do not have the target character.

There were 15 trials with the subject focusing on a particular character. This lead to a total of 180 intensifications (i.e., 12 times 15) for a single character. After the 180th intensification, there was an interval for the subject to prepare to focus on the next character of the target word. This interval lasted for $2.5 \mathrm{~s}$. Each VERP response was stored as a 240 data point signal (i.e., $1 \mathrm{~s}$ in length) where the onset of the intensification denotes the beginning of the signal.

The three sessions that were used here, session 10 , 11 and 12 had 5, 6 and 8 runs, respectively. As each run represented a target word and each word contained three to five characters; there were a total of 19, 23 and 30 words available for our experimental study.

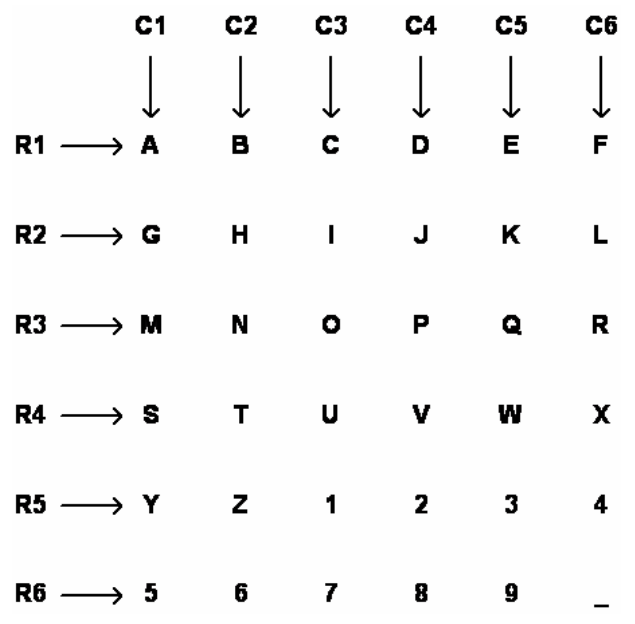

Fig. 1: Spelling paradigm matrix ${ }^{[4]}$

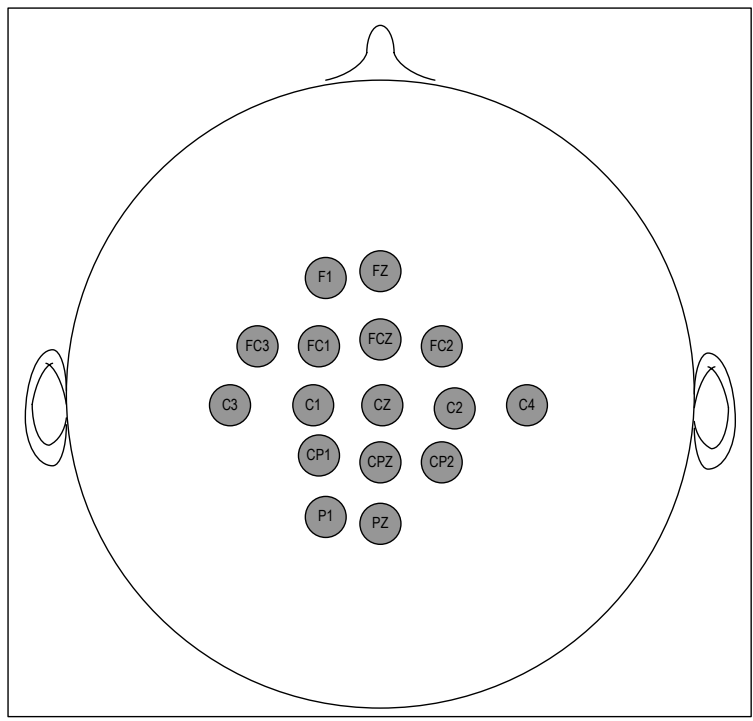

Fig. 2: Positions of the selected electrodes

Signals from sixteen channels from locations $\mathrm{Cz}$, $\mathrm{Pz}, \mathrm{Fcz}, \mathrm{Fz}, \mathrm{C} 1, \mathrm{Cp} 1, \mathrm{Cpz}, \mathrm{C} 2, \mathrm{Cp} 2, \mathrm{C} 3, \mathrm{FC} 3, \mathrm{P} 1, \mathrm{Fc} 1$, $\mathrm{Fc} 2, \mathrm{~F} 1, \mathrm{C} 4$ were taken for consideration in the analysis. These channels were found to be suitable after some preliminary signal analysis by plotting their amplitude responses in the P300 latency window; this is in line with a previous report ${ }^{[8]}$. There was a stimulus code that represented the actual column or row that was being intensified that was stored as a reference.

\section{MATERIALS AND METHODS}

Single trial over averaging: To find one single character using the paradigm, there will be 12 random 
intensifications of each row and column as mentioned previously. The signal recorded during the intensification of a column or a row that contains the specific letter will be known as the target signal while the signal recorded during the intensification of a column or a row that does not contain the specific letter will be known as the non-target signal. Therefore, for one character identification, all the 6 rows and 6 columns will be intensified once and this results in two target signals and 10 non-target signals to be recorded.

This procedure is repeated for the same character for a further 14 times, giving in total $12 \mathrm{H} 15=180$ signal representations for a character. Out of these, 30 would be target signals and 150 would be the non-target signals.

In the popular averaging method, the corresponding 15 signals from each row and column will be averaged to obtain two signals. This is performed to reduce the background EEG and thereby enhancing the VERP responses. However, this procedure demands a higher level of resources like time, storage, computational complexity, etc. More importantly the individual VERP responses vary from each other (due to non-strict time locking) and the averaged signal that is represented obtained by ensemble averaging may not reflect the original VERP components in all their characteristics and resemblances.

As a result, it is useful to analyse VERP responses from single trials in order to reduce the response times and other resources. This has become a challenge to many researchers and PCA is one of the techniques that have been used for this purpose ${ }^{[7]}$.

Recently, a method that predicted all the characters correctly in this dataset except a single letter $\mathrm{G}$ (from run 2 in sessions 11 that was detected as $H$ ) was reported ${ }^{[8]}$. However, this was achieved through a multitude of complex methods that involved some form of averaging, PCA, temporal and spatial manipulations of independent components that were considered with back projection analysis. In this study, we propose methods based on the de facto PCA, without any other manipulations of the raw signals. Specifically, our methods select the suitable PCs in the hope to increase the detection of the intended (target) character using only a single trial data. In the experimental study, we tested the suitability of our methods in correctly predicting the intended letter and comparing these with the other standard PC selection methods.

Principal component analysis: Principal component analysis finds a linear transformation of a data set that maximises the variance of the transformed variables subject to orthogonality constraints on the transformation and transformed variables.

Standard PCA method ${ }^{[9]}$ to extract single trial VERP components from the recorded signals was carried out. The covariance of the recorded signal (that contains both VERP and background EEG), $\mathrm{W}$ was computed using

$$
\mathrm{R}=\mathrm{E}\left(\mathrm{W}^{\mathrm{T}} \mathrm{W}\right)
$$

$\mathrm{R}$ would be a matrix of size $16 \mathrm{Hl} 6$ because of the size of matrix W that was $240 \mathrm{Hl} 6$ (the length of one recorded signal was 240 and there were 16 channels). Let $F$ be the orthogonal matrix of eigen vectors of $R$ and $\mathrm{D}$ as the diagonal matrix of its eigenvalues, then $\mathrm{D}=\operatorname{diag}\left(\mathrm{d}_{1} \ldots \mathrm{d}_{\mathrm{n}}\right)$. There were 16 set of eigenvalues and eigenvectors.

Then the PCs (dimension of $240 \mathrm{Hl} 16$ ) could be computed using,

$$
\mathrm{Y}=\mathrm{F}^{\mathrm{T}} \mathrm{W}^{\mathrm{T}}
$$

Some of the PCs will represent VERP and some will represent background EEG. The selections of PCs from all PCs were carried out by 4 methods, namely RP, KSR, SPR and SSPR. The first two methods are the standard ones used in $\mathrm{PCA}^{[9]}$.

These selected PCs were then used in reconstruction, where the reconstructed signal now contains only the VERP. The reconstruction was done using

$$
\mathrm{X}=\mathrm{FF}^{\mathrm{T}} \mathrm{YY}^{\mathrm{T}} \text {. }
$$

where the FF and $\mathrm{YY}$ corresponds to the selected eigenvectors and PCs. Note that the dimension of the reconstructed VERPs would still be $240 \mathrm{H} 16$.

Different methods of principal component selection residual power: In the RP method ${ }^{[8]}$, the first or first few PCs were selected where the percentages of corresponding eigenvalues were $95 \%$ over the total eigenvalues:

$$
\sum e_{r p} / \sum e \geq 0.95
$$

where $\mathrm{e}_{\mathrm{rp}}$ and e represent the selected eigenvalues and the set of all the eigenvalues, respectively. The remaining PCs were omitted and only the selected PCs 
were used for the reconstruction of the VERP signals. The value $95 \%$ was chosen as it is the common rule of thumb measure.

Kaiser method: KSR method ${ }^{[9]}$ selects the PCs such that the eigenvalues of the selected PCs were more than 1.0 :

$$
e_{k s r} \geq 1.0
$$

where $e_{\mathrm{ksr}}$ represents the selected eigenvalues. The PCs corresponding to the discarded eigenvalues were omitted and only the selected PCs were used for the reconstruction of the VERP signals.

Spectral power ratio: The SPR method is specifically suited for the extractions of single trial VERP under heavy noise/EEG contamination ${ }^{[10]}$ since the VERP is found buried in the ongoing EEG activity whose spectral power is very high compared to the VERP. The SER method ${ }^{[12]}$ we used for this purpose needs visual observation and hence not suitable for online applications. But SPR is suitable here since we are interested in P300 responses, this method would be adjusted to select only the PCs that contain significant amount of $0-8 \mathrm{~Hz}$ spectral energy. This frequency limit could be varied according to the purpose (in our case, P300 responses are generally limited to $8 \mathrm{~Hz}^{[11]}$ ). We used a low pass filter with the combination of a 9th order forward and 9th order reverse Butterworth digital filter with a cut-off frequency at $8 \mathrm{~Hz}$. A minimum attenuation of $30 \mathrm{~dB}$ was achieved in the stop band, with the transition band being between 8 and $12 \mathrm{~Hz}$. The reason for both forward and reverse filtering was to ensure that there would be no phase distortion.

The significant amount of $0-8 \mathrm{~Hz}$ spectral energy is determined using a specific threshold:

$$
\begin{array}{ccc}
\mathrm{PCspr}=\mathrm{PC} & \text { if } & \mathrm{PC}_{0-8 \mathrm{~Hz}} / \mathrm{PC}^{3} \mathrm{t} \\
\mathrm{PCspr}=0 & \text { if } & \mathrm{PC}_{0-8 \mathrm{~Hz}} / \mathrm{PC}<\mathrm{t}
\end{array}
$$

Following some experimental simulations, we found that any value in the range 0.4-0.45 were sufficient as thresholds, i.e., for the PC under consideration, if the ratio of spectral power below $8 \mathrm{~Hz}$ over the total spectral power exceeded this threshold, then that PC would be retained for reconstruction. The other PCs with SPR below this threshold were set to zero. Next, these selected PCs were used to reconstruct the VERP signals. In this experiment, we have chosen 0.4 as the threshold throughout but it should be noted that this threshold $t$ is likely to be subject dependant.
Sandwich spectral power ratio: The SSPR method is similar to SPR method except that we introduced an additional low pass filter in between two levels of PCASPR applications. In other words, after reconstruction using PCA-SPR method, the VERP signals are again filtered using this Butterworth filter. Next, PCA-SPR method is applied again to these filtered signals. However, the threshold value of the later SPR is slightly reduced in the range of $0.2-0.3$ (we used 0.2 through out) to take in account for the fact that a lesser degree of background EEG exists. The inserted Butterworth filter had the same specifications as the one used in SPR.

\section{PRELIMINARY TEST FOR CONTRASTING P3 RESPONSES}

We conducted a preliminary experiment (using a subset of the data) to test the efficiencies of the standard PC selection methods, by using a set of fifteen trials from a single character chosen randomly. Out of the two target signals and ten non-target signals (from each trial), we picked up the P300 component as the most positive peak with latency of $290-340 \mathrm{~ms}$ since the P300 peak latency varies with respect to task complexity. The time window we selected here for P300 detection was from 290 to $340 \mathrm{~ms}$, since the training set of data from session 10 and session 12 confirms this as all the P300 components from these sessions were inside this window. However, this could be subject (data) dependant and may need to be relaxed in other cases.

We obtained higher target amplitudes as compared to some of the non-target amplitudes. Nevertheless, we still found the presence of some non-target amplitudes that were comparable to the target amplitudes.

We repeated this experiment using proposed SPR and SSPR methods and the results of this experiment are as reported as the best target and best non-target peaks in Table 1 and 2. The P300 amplitude was measured with reference to zero. We report the averages of the P300 amplitudes (after PCA selection methods) from 3rd, 5th, 10th, 11th and 15th trials. Instead of reporting results from all 15 trials, we chose to report the averaged results from 5 trials to save space. And rather than choosing 5 trials randomly, we chose the best 5 trials for each target and non-target cases. We opted for this approach as this would reflect a proper evaluation of the performances of the methods i.e., an increased reliability of our proposed methods).

Even though the feat of RP and KSR were similar in most of the target trials (as they selected the same $\mathrm{PCs})$, there were slight variations in non-target trials 
Am. J. Applied Sci., 5 (6): 639-644, 2008

Table 1: Comparison of target P300 amplitudes using RP, KSR, SPR and SSPR methods

\begin{tabular}{lllll}
\hline No. of trials & RP & KSR & SPR & SSPR \\
\hline 3 & 7.65 & 7.65 & 7.75 & 7.92 \\
5 & 7.62 & 7.62 & 7.70 & 7.93 \\
10 & 8.17 & 8.17 & 8.21 & 8.33 \\
11 & 8.07 & 8.07 & 8.12 & 8.34 \\
15 & 7.95 & 7.95 & 8.00 & 8.14 \\
\hline
\end{tabular}

Table 2: Comparison of non -target P300 amplitudes of RP, KSR, SPR and SSPR methods

\begin{tabular}{lllll}
\hline No. of trials & RP & KSR & SPR & SSPR \\
\hline 1 & 0.00 & 0.97 & 0.81 & 0.00 \\
7 & 1.53 & 1.44 & 1.54 & 1.38 \\
8 & 1.03 & 1.03 & 1.19 & 0.00 \\
11 & 2.49 & 2.49 & 2.10 & 0.00 \\
15 & 1.29 & 1.29 & 1.26 & 0.34
\end{tabular}

Zero amplitude denotes that no P300 peak was detected in the chosen time window

where RP was found to be inferior to KSR. As we found the performance of the RP method to be poor, we excluded this method from further analysis and comparisons.

\section{TARGET WORD PREDICTION AND RESULTS}

In this part of the experimental study, we predicted the character in a word by detecting the extracted P300 amplitudes for each row and column (again as the most positive peak in the range of 290 to $340 \mathrm{~ms}$ ). The character was assumed to be correctly predicted if the following condition was satisfied. After applying PCA and averaging from 16 channels, if the P300 amplitude of target row was higher than non-target rows and if the P300 amplitude of target column was higher than nontarget columns. This process was repeated for all 15 trials and for the number of characters in the word and the number of correct predictions was identified.

Table 3 reports the number of correct predictions of applying SSPR to a few randomly chosen characters from 15 trials. This was then applied to all the characters in every word from the three sessions and the total numbers of correct predictions are as listed in Table 4. Note that the total possible correct predictions in sessions 10,11 and 12 were 285,345 and 450 , respectively.

\section{DISCUSSION}

From Table 3, it can be seen that the number of correct predictions using SSPR were higher than SPR and KSR. We also conducted statistical t-test of all the target P300 amplitudes obtained using KSR, SPR and SSPR methods from all the characters. The results of P300 amplitudes from target rows indicate that
Table 3: Comparison of correct predictions of target characters by KSR, SPR and SSPR methods

\begin{tabular}{lccc}
\hline & Correct predictions out of 15 trials & \\
Target character & KSR & SPR & SSPR \\
\hline D & 7 & 12 & 13 \\
R & 10 & 11 & 11 \\
G & 6 & 7 & 9 \\
H & 10 & 11 & 13 \\
A & 12 & 13 & 14 \\
O & 12 & 13 & 15 \\
Total & 57 & 67 & 75 \\
\hline
\end{tabular}

Table 4: Comparison of total correct predictions of target characters by KSR, SPR and SSPR methods

\begin{tabular}{llll}
\hline Sessions & KSR & SPR & SSPR \\
\hline Session 10 & 191 & 236 & 269 \\
Session 11 & 212 & 231 & 288 \\
Session 12 & 301 & 363 & 390 \\
Total & 704 & 830 & 947 \\
\hline
\end{tabular}

amplitudes from SPR is significantly higher than amplitudes from $\mathrm{KSR}(\mathrm{p}=2.5 \mathrm{E}-4)$ while amplitudes from SSPR is higher than SSR $(p=0.0001)$. The results of P300 amplitudes from target columns indicate that amplitudes from SPR is also significantly higher than amplitudes from KSR (1.04 E-6) but amplitudes from SSPR are only marginally significant from SPR $(p=$ 0.2525). Overall, from the statistical results, we can conclude that SSPR gives better results than SPR, which in turn is better than KSR.

\section{CONCLUSION}

The VERP based BCI typically depends on the accurate detection of P300 peak amplitudes. Enhancement of signal quality by our proposed SPR and SSPR methods to select the PCs has shown an improvement in the target character prediction. SSPR method gave better performance than SPR method, but both SSPR and SPR performed better than RP and KSR methods.

Based on the results, we conclude that our proposed SSPR technique would be suitable and considered as an enhancement in single trial analysis using PCA for VERP extraction. Specifically, the method gave improved performance in target character prediction using P300 amplitudes in the specific time window. The characteristics of SSPR in reducing the background EEG helps to enhance differences in the target and non-target signals and this would prove to be useful in VERP based BCI application. It is possible to extend the SSPR method for any other event-related response analysis as long as the frequency range of the concerned parameter is known. 


\section{ACKNOWLEDGMENT}

The authors wish to acknowledge the Wadsworth Center, New York State Department of Health, Albany, NY, USA for their P300 evoked potential data set IIb that was placed in the BCI Competition 2003 website.

\section{REFERENCES}

1. Wolpaw, J.R., N. Birbaumer, D.J. McFarland, G. Pfurtscheller and T.M. Vaughan, 2002. Braincomputer interfaces for communication and control. Clin. Neurophysiol., 113: 767-791.

2. Palaniappan, R., 2006. Utilizing gamma band spectral power to improve mental task based brain computer interface design. IEEE Trans. Neural Syst. Rehabilitation Eng., 14 (3): 1-6.

3. Mensh, B.D., J. Werfel and H.S. Seung, 2004. BCI competition 2003-data set Ia: Combining gammaband power with slow cortical potentials to improve single-trial classification of electroencephalographic signals. IEEE Trans. Biomed. Eng., 51 (6): 1052-1056.

4. Donchin, E., K.M. Spencer and R. Wijesinghe, 2000. The mental prosthesis: Assessing the speed of a P300 based brain-computer interface. IEEE Trans. Rehabilitation Eng., 8 (2): 174-179.

5. Wang, Y., R. Wang, X. Gao, B. Hong and S. Gao, 2006. A practical VEP based brain-computer interface. IEEE Trans. Neural Syst. Rehabilitation Eng., 14 (2): 234-239.
6. Aunon, J.I., C.D. McGillem and D.G. Childers, 1981. Signal processing in event potential research: averaging and modelling. CRC Criti. Rev. Bioeng., 5: 323-367.

7. Lange, D.H. and G.F. Inbar, 1996. Variable singletrial evoked potential estimation via principal component identification. Proceedings of 18th IEEE EMBS Ann. Int. Conf., pp: 954-955.

8. Xu, N., X. Gao, B. Hong, X. Miao, S. Gao and F. Yang, 2004. BCI competition 2003-dataset IIb: Enhancing P300 wave detection using ICA-based subspace projections for BCI applications. IEEE Trans. Biomed. Eng., 51 (6): 1067-1072.

9. Jolliffe, I.T., 1986. Principal Component Analysis, New York.

10. Andrews, S., R. Palaniappan and V.S. Asirvadam, 2004. Single trial source separation of VEP signals using selective principal components. Proceedings of 2nd International Conference on Advances in Medical Signal and Information Processing, Malta G.C., Europe, pp: 51-57.

11. Polich, J., 1991. P300 in clinical applications: Meaning, method and measurement. Am. J. EEG Technol., 31: 201-231.

12. Andrews, S., R. Palaniappan and N. Kamel, 2005. Single Trial VEP Source Separation by Selective Eigen RatePrincipalComponents. 5th International Enformatika Conference 2005, Prague, Czech Republic. 